\title{
Association Between Admission Serum Phosphate and Risk of Acute Kidney Injury in Critically III Patients with Rhabdomyolysis: A Retrospective Study Based on MIMIC-III
}

\section{Tao Wen}

General Hospital of People's Liberation Army: Chinese PLA General Hospital https://orcid.org/00000002-6944-0739

\section{Zhi Mao}

General Hospital of People's Liberation Army: Chinese PLA General Hospital

Chao Liu

General Hospital of People's Liberation Army: Chinese PLA General Hospital

\section{Xiaoli Wang}

General Hospital of People's Liberation Army: Chinese PLA General Hospital

Feihu Zhou ( $\square$ feihuzhou301@126.com )

The First Medical Centre, Chinese PLA General Hospital https://orcid.org/0000-0001-6154-013X

\section{Research}

Keywords: Rhabdomyolysis, acute kidney injury, critical care medicine, intensive care unit, serum phosphate, MIMIC- $\rrbracket$

Posted Date: November 15th, 2021

DOI: https://doi.org/10.21203/rs.3.rs-1063939/v1

License: (a) (1) This work is licensed under a Creative Commons Attribution 4.0 International License. Read Full License 


\section{Abstract}

Background The incidence of acute kidney injury(AKI) is high in critically ill patients with rhabdomyolysis. Limited evidence was proved of the association between serum phosphate levels at intensive care unit(ICU) admission and the subsequent risk of AKI. Our study aims to assess if serum phosphate level at admission was independently associated with AKI risk in these patients.

Methods This study extracted and analyzed data from Medical Information Mart for Intensive Care邓(MIMIC-Z,version1.4). Rhabdomyolysis was defined as a peak creatine kinase(CK) level higher than 1000 $\mathrm{U} / \mathrm{L}$. Serum phosphate was measured within the first day into the ICU and was categorized to 4 groups(<2.6, 2.6-3.4, 3.5-4.5, >4.5mg/dl). AKI was defined according to the Kidney Disease Improving Global Outcome (KDIGO) guidelines. Adjusted smoothing spline plots and multivariate logistic regressions were carried out to explode the association between serum phosphate and risk of AKI. Subgroup analyse was applied to verify the consistency of the association.

Results Three hundred and twenty-one patients(67.8\% male) diagnosed as rhabdomyolysis were eligible for this analysis. AKI occurred in 204(63.6\%) patients of total. Incidence of AKI with admission serum phosphate groups $<2.6,2.6-3.4,3.5-4.5$ and $>4.5 \mathrm{mg} / \mathrm{dl}$ were $52.6 \%, 56.8 \%, 68.4 \%$ and $75.9 \%$, respectively. Smoothing spline curve showed that there was a positive curve between the elevated phosphate values and increasing risk of $\mathrm{AKI}$, and there was no threshold saturation effect. In multivariate logistic regression, OR was $1.3(95 \% \mathrm{Cl} 1.1-1.6, \mathrm{P}=0.012, \mathrm{P}$ trend=0.034) after adjusting confounders. Subgroup analyses proved the consistency of the relationship in these patients except in the strata of creatine kinase.

Conclusion In rhabdomyolysis patients admitted to ICU, serum phosphate level at admission was independently associated with an increased risk of AKI. As phosphate levels rise, the risk of AKI increased.

\section{Introduction}

Rhabdomyolysis is a kind of muscular disease characterized by muscle damage that results in the leakage of muscle cell contents which include electrolytes, enzymes and myoglobin[1]. When these constituents were released from injured cells into the circulation, physiological functions become disordered and organs suffer damage due to the toxic effects[2]. Rhabdomyolysis is known to have a variety of causes, including strenuous exercise, trauma, tissue compression, surgery, heat stroke, alcoholism, drug intoxication, infection and so on[2, 3]. Rhabdomyolysis can be caused by a number of genetic diseases, which usually appear in childhood, such as sickle cell anemia and myopathy, resulting in enzyme deficiency and inadequate ATP transmission $[4,5]$. Rhabdomyolysis due to the novel coronavirus has been detected as a result of the spread of COVID-19 in the past two years[6, 7]. The typical triad of rhabdomyolysis includes muscle pain, muscle weakness and tawny urine, but it has been 
reported that the triad is only observed in less than $10 \%$ of patients and some patients only have asymptomatic increase in serum enzyme level[ $[8,9]$.

Acute kidney injury(AKI) is one of the most severe complications of rhabdomyolysis. It is estimated that about $13 \%-50 \%$ of patients develop AKI after rhabdomyolysis[10-13]. The pathology of AKI secondary to rhabdomyolysis was first reported during World War II, when four cases of crush injury were reported, characterized by diffuse tubular injury with hyperpigmentation followed by renal dysfunction[14]. The mechanisms behind rhabdomyolysis induced AKI are complex. Previous research indicates that these mechanisms may include renal vascular stenosis, ischemic tubular injury and tubular obstruction caused by free myoglobin $[5,9,15,16]$.

Phosphorus exists in two forms in the blood, organic phosphorus and inorganic phosphorus. Organophosphates and phospholipids are present in large amounts in blood cells and plasma. Blood phosphorus usually refers to inorganic phosphorus in plasma. Serum phosphate is the item we usually measure, which can reflect the inorganic phosphorus metabolism in the blood[17, 18]. In patients with moderate or severe kidney disease, a higher level of serum phosphate values is commonly seen. Besides, they are correlated with calcification of heart valves and blood vessels, which may result in an increase in cardiovascular events and mortality $[19,20]$. Kidney excretion of phosphorus is the key to maintain the balance of blood phosphate. Thus, on one hand, hyperphosphatemia is common in patients with $\mathrm{AKI}$, and on the other hand, hyperphosphatemia could cause AKI itself through acute phosphate nephropathy(APN)[21]. The importance of phosphate are becoming emphasized in patients with rhabdomyolysis. At present, the evidence about the relationship between phosphate and AKI is still insufficient, and there is still a lack of a large number of studies. Therefore, our study focused on the correlation between phosphate values and risk of AKI in rhabdomyolysis patients admitted to the ICU.

\section{Methods}

2.1 The Database. The data was acquired from Medical Information Mart for Intensive Care(MIMICQ,version1.4), which is a open and public online database. From 2001 to 2012, it collected clinical information on more than 58,000 ICU inpatient visits from 38,645 non-newborn patients and 7875 newborn patients admitted to Beth Israel Deaconess Medical Center, USA[22]. After finishing the National Institutes of Health's web-based course and the Protecting Human Research Participants exam (no. 29493483), database permission was authorized. The Institutional Review Boards of Beth Israel Deaconess Medical Center (Boston, MA) and the Massachusetts Institute of Technology (Cambridge, MA) approved the project. All patient information is hidden to protect their privacy. We extracted baseline demographics and laboratory data of the patients from database in this study. 
2.2 Population Selection. All recruited patients were adult patients with age over eighteen years old. The diagnosis of rhabdomyolysis was based on the 9th International Classification of Diseases (ICD-9) criteria in this study. Rhabdomyolysis was defined according to the peak value of creatine kinase(CK) after admission which was over $1000 \mathrm{U} / \mathrm{L}[2,23,24]$. Only those who were at first admission for ICU and had been hospitalized for more than two days were admitted into our study.

2.3 Data Extraction. Data of the patients was extracted through Structured Query Language from MIMICه. Following data was extracted or calculated: vital signs, patient demographics, lab parameters, comorbidities and scoring systems. Baseline data were obtained from patients admitted to the ICU within 24 hours. The following laboratory parameters were extracted: phosphate, creatine kinase(CK), creatine $(\mathrm{Cr})$, blood urea nitrogen(BUN), biocarbonate, hematocrit(HCT), alanine transaminase(ALT), red cell distribution width(RDW), aspartate aminotransferase(AST), hemoglobin(HGB), white blood cell(WBC), calcium, sodium, potassium, lactate and platelet. The comorbidities included hypertension, diabetes, atrial fibrillation(AF), acute heart failure (AHF), acute respiratory distress syndrome (ARDS), acute liver failure(ALF) and stroke. We also extracted age, gender, ethnicity, mean blood pressure (MBP), mean heart rate(HR), mean respiratory rate(RR), mean temperature, mean SPO2, renal replacement therapy(RRT), and length of stay (LOS) in hospital. Sequential organ failure assessment (SOFA) score and acute physiology score $₫($ APS $\otimes)$ were calculated for each patient.

2.4 Outcomes. The primary exposure was the serum phosphate value, which is a common indicator, was defined as an initial phosphate value measured within $24 \mathrm{~h}$ of admission to the ICU. AKI occurring after ICU admission was the primary outcome in this study. The classification of AKI was determined by Kidney Disease Improving Global Outcomes (KDIGO)[25]. An absolute increase in $\mathrm{SCr}$ of $\geq 0.3 \mathrm{mg} / \mathrm{dL}$ within 48 hours or $\geq 1.5$ times baseline value within 7 days after admission date was used to define AKI[26].

2.5 Statistical Analysis. The measurement data were presented as mean \pm standard deviation(SD) or medians, inter quartile range(IQR). They were compared by ANOVA test. The count data were expressed as percentage. Chi-square test was applied to compare between groups. In case of non-normal distribution or variance heterogeneity, non-parametric test was applied between groups, which included Mann-Whitney $U$ test and Kruskal-Wallis test. Baseline characteristics of the patients were stratifed by four phosphate groups( $<2.6,2.6-3.4,3.5-4.5$ and $>4.5 \mathrm{mg} / \mathrm{dl})$ based on its distribution. For comparison between groups, group of lowest incidence of AKI (phosphate $<2.6 \mathrm{mg} / \mathrm{dl}$ ) was chosen as the reference.

We explored the non-linear relationship between AKI and phosphate through a generalized additive model (GAM) with an adjustment for potential confounders. A smoothing spline plot was created. Then we used a multivariate logistic regression model to ascertain whether there was a significant association between AKI and phosphate. Non-adjusted and adjusted odds ratio(OR) as well as 95 percent confidence interval $(95 \% \mathrm{Cl})$ were applied to present the results. Two different kinds of adjusted multivariate models were created according to the four phosphate groups for each endpoint. Variables were chosen as potential confounders based on epidemiological and biological background. After that these confounders were used to generate the adjusted models based on changes of statistical effect estimation over 10 percent[27]. In model I, age and gender were adjusted as conventional variables. In model II, more 
meaningful variables were added for adjustment including age, gender, ethnicity, CK, CR, BUN, calcium, potassium, HCT, bicarbonate, RDW, hypertension, AF, stroke, SOFA score and APS $₫$ score. To perform tests for liner trend, we entered the median value of each level of phosphate in the models as a continuous variable. To explode the effect of phosphate differing across various subgroups, subgroup analyses were performed. All subgroups were classifed by age, gender, MAP, HR, RR, Temperature, SPO2, CK, CR, BUN, ALT, AST, bicarbonate, HCT, HGB, calcium, sodium, potassium, WBC, lactate, platelet, hypertension, diabetes, AHF, ALF, AF, stroke, SOFA score and APS $₫$ score. Multiplicative interactions were estimated by adding interaction terms according to the likelihood ratio test.

All data were analyzed with EmpowerStats software version 2.2 (X\&Y solutions, Inc., Boston, MA) and R sofware(version 3.6). Two-sided $P \otimes 0.05$ was considered to be statistically significant.

\section{Results}

3.1 Subject Characteristics According to the admission criteria, three hundred and twenty-one adult patients met the criteria of this study. The screening flow chart is shown in Figure 1.

Baseline data of the patients are shown in Table 1. Based on the values of serum phosphate on admission, all enrolled patients were divided into four groups equally: $<2.6 \mathrm{mg} / \mathrm{dl}$, 78 patients(24.2\%); 2.6 $3.4 \mathrm{mg} / \mathrm{dl}, 81$ patients $(25.2 \%) ; 3.5-4.5 \mathrm{mg} / \mathrm{dl}, 79$ patients( $24.5 \%)$ and $>4.5 \mathrm{mg} / \mathrm{dl}, 83$ patients $(25.8 \%)$, respectively. Of the 321 patients, the mean age was $53.8 \pm 16.9$ years. $217(67.6 \%)$ patients were male and 104 (32.4\%) were female. 221(68.8\%) patients were white people and black people $42(13.1 \%)$ were black people. Hispanic and asian people maked up only a small percentage. Further comparison were made among the four different groups. The result showed that patients with higher phosphate levels had older age, higher values of mean SPO2, CK, CR, BUN, AST, potassium, lactate and had lower mean temperature and lower calcium, the p-value was less than 0.05. Moreover, patients with higher phosphate levels tended to have higher SOFA score and APS $\triangle$ score, as well as higher probability of receiving RRT treatment than other patients.

3.2 Incidence of AKI AKI occurred in 204 patients(63.6\%) with rhabdomyolysis. The incidence of AKI was $52.6 \%, 56.8 \%, 68.4 \%$ and $75.9 \%$ with phosphate groups $<2.6,2.6-3.4,3.5-4.5$ and $>4.5 \mathrm{mg} / \mathrm{dl}$, respectively. Patients with phosphate $>4.5 \mathrm{mg} / \mathrm{dl}$ were found to have the highest AKI incidence, with the association preserved for all stages of AKI (Table 1). The characteristics and their comparison with or without AKI are

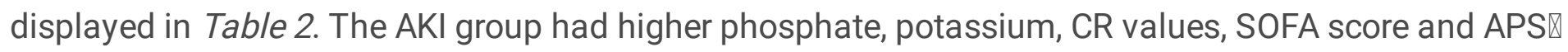
score. They also tended to have AF, to use RRT than non-AKI group and to stay longer in hospital. No differences were observed in most variables between the AKI group and non-AKI gourp(age, gender, ethnicity, MAP, HR, RR, temperature, SPO2, CK, BUN, ALT, AST, platelet, bicarbonate, HCT, HGB, calcium, sodium, potassium, lactate, WBC, RDW, hypertension, diabetes, AHF, ALF, ARDS, stroke, p-value>0.05). 3.3 Characteristics between AKI stage groups We further compared the characteristics of the patients among three stages of AKI (Table 3). Of the 204 patients who developed AKI, 46 patients were in stage 1 , 76 patients were in stage 2 and 82 patients were in stage 3 . The trend of each stage with the elevated phosphate levels was shown in a line chart in Figure 2. Incidence of AKI showed no single upward or downward trend in stage 1 and stage 2. In stage 1, it presented a gradual decline. Stage 2 showed a trend 
of rising and then falling, the highest incidence occurred in the second group with phosphate 2.6$3.4 \mathrm{mg} / \mathrm{dl}$. Stage 3 showed an obvious upward trend with the increase of phosphate levels. Patients of phosphate more than $4.5 \mathrm{mg} / \mathrm{dl}$ in stage 3 had the highest incidence of $\operatorname{AKI}(12.1 \%)$. Patients from stage 3 also had higher $\mathrm{CK}, \mathrm{CR}, \mathrm{BUN}$, bicarbonate, potassium values, SOFA score and APS $₫$ score and lower calcium values than the other two stages. ALF, AF and use of RRT were more easily to discover in the stage 3 group. 
Table 1

Characteristics of the study population based on four phosphate groups

\begin{tabular}{|c|c|c|c|c|c|c|}
\hline \multirow[t]{2}{*}{ Characteristics } & \multirow[t]{2}{*}{ All } & \multicolumn{4}{|c|}{ phosphate levels (mg/dL) } & \multirow[t]{2}{*}{$\mathbf{P}$} \\
\hline & & $<2.6$ & $2.6-3.4$ & $3.5-4.5$ & $>4.5$ & \\
\hline$N$ & 321 & 78 & 81 & 79 & 83 & \\
\hline Age(year) & $\begin{array}{l}53.8 \pm \\
16.9\end{array}$ & $\begin{array}{l}49.1 \pm \\
16.6\end{array}$ & $\begin{array}{l}55.2 \pm \\
18.3\end{array}$ & $\begin{array}{l}53.8 \pm \\
17.2\end{array}$ & $\begin{array}{l}56.8 \pm \\
14.6\end{array}$ & 0.026 \\
\hline Gender(n,\%) & & & & & & 0.97 \\
\hline male & $\begin{array}{l}217 \\
(67.6 \%)\end{array}$ & $\begin{array}{l}51 \\
(65.4 \%)\end{array}$ & $\begin{array}{l}55 \\
(67.9 \%)\end{array}$ & $\begin{array}{l}54 \\
(68.4 \%)\end{array}$ & $\begin{array}{l}57 \\
(68.7 \%)\end{array}$ & \\
\hline female & $\begin{array}{l}104 \\
(32.4 \%)\end{array}$ & $\begin{array}{l}27 \\
(34.6 \%)\end{array}$ & $\begin{array}{l}26 \\
(32.1 \%)\end{array}$ & $\begin{array}{l}25 \\
(31.6 \%)\end{array}$ & $\begin{array}{l}26 \\
(31.3 \%)\end{array}$ & \\
\hline Ethnicity(n,\%) & & & & & & 0.623 \\
\hline asian & $4(1.2 \%)$ & $1(1.3 \%)$ & $3(3.7 \%)$ & $0(0.0 \%)$ & $0(0.0 \%)$ & \\
\hline hispanic & $11(3.4 \%)$ & $1(1.3 \%)$ & $4(4.9 \%)$ & $4(5.1 \%)$ & $2(2.4 \%)$ & \\
\hline black & $\begin{array}{l}42 \\
(13.1 \%)\end{array}$ & $\begin{array}{l}10 \\
(12.8 \%)\end{array}$ & $9(11.1 \%)$ & $\begin{array}{l}13 \\
(16.5 \%)\end{array}$ & $\begin{array}{l}10 \\
(12.0 \%)\end{array}$ & \\
\hline white & $\begin{array}{l}221 \\
(68.8 \%)\end{array}$ & $\begin{array}{l}55 \\
(70.5 \%)\end{array}$ & $\begin{array}{l}54 \\
(66.7 \%)\end{array}$ & $\begin{array}{l}53 \\
(67.1 \%)\end{array}$ & $\begin{array}{l}59 \\
(71.1 \%)\end{array}$ & \\
\hline other & $\begin{array}{l}43 \\
(13.4 \%)\end{array}$ & $\begin{array}{l}11 \\
(14.1 \%)\end{array}$ & $\begin{array}{l}11 \\
(13.6 \%)\end{array}$ & $9(11.4 \%)$ & $\begin{array}{l}12 \\
(14.5 \%)\end{array}$ & \\
\hline $\operatorname{AKI}(\mathrm{n}, \%)$ & $\begin{array}{l}204 \\
(63.6 \%)\end{array}$ & $\begin{array}{l}41 \\
(52.6 \%)\end{array}$ & $\begin{array}{l}46 \\
(56.8 \%)\end{array}$ & $\begin{array}{l}54 \\
(68.4 \%)\end{array}$ & $\begin{array}{l}63 \\
(75.9 \%)\end{array}$ & 0.008 \\
\hline $\mathrm{MAP}(\mathrm{mmHg})$ & $\begin{array}{l}82.0 \pm \\
12.1\end{array}$ & $\begin{array}{l}84.3 \pm \\
13.1\end{array}$ & $\begin{array}{l}81.9 \pm \\
13.0\end{array}$ & $81.4 \pm 9.5$ & $\begin{array}{l}80.4 \pm \\
12.5\end{array}$ & 0.223 \\
\hline $\mathrm{HR}(/ \min )$ & $\begin{array}{l}92.7 \pm \\
15.4\end{array}$ & $\begin{array}{l}94.6 \pm \\
16.0\end{array}$ & $\begin{array}{l}92.5 \pm \\
16.4\end{array}$ & $\begin{array}{l}91.8 \pm \\
13.6\end{array}$ & $\begin{array}{l}92.2 \pm \\
15.5\end{array}$ & 0.674 \\
\hline $\mathrm{RR}(/ \min )$ & $19.7 \pm 3.8$ & $19.7 \pm 3.8$ & $19.4 \pm 3.7$ & $19.8 \pm 3.4$ & $20.0 \pm 4.4$ & 0.756 \\
\hline Temperature $\left({ }^{\circ} \mathrm{C}\right)$ & $37.0 \pm 0.7$ & $37.3 \pm 0.8$ & $37.1 \pm 0.6$ & $37.0 \pm 0.7$ & $36.8 \pm 0.6$ & 0.002 \\
\hline SP02(\%) & $96.9 \pm 3.4$ & $97.3 \pm 1.6$ & $96.9 \pm 4.0$ & $96.0 \pm 4.8$ & $97.4 \pm 1.8$ & 0.034 \\
\hline
\end{tabular}

AKl:acute kidney injury;CK:creatine kinase; CR:creatine; BUN:blood urea nitrogen; ALT:alanine transaminase; AST:aspartate aminotransferase; HCT:hematocrit; HGB:hemoglobin; WBC:white blood cell; RDW:red cell distribution width; AHF:acute heart failure; ALF:acute liver failure; AF:atrial fibrillation; ARDS:acute respiratory distress syndrome; MBP:mean blood pressure (MBP); HR:heart rate; RR:respiratory rate; RRT:renal replacement therapy; LOS:length of stay; SOFA:Sequential organ failure assessment; APS $₫$ :acute physiology score $\rrbracket$. 


\begin{tabular}{|c|c|c|c|c|c|c|}
\hline \multirow[t]{2}{*}{ Characteristics } & \multirow[t]{2}{*}{ All } & \multicolumn{4}{|c|}{ phosphate levels (mg/dL) } & \multirow[t]{2}{*}{$\mathbf{P}$} \\
\hline & & $<2.6$ & $2.6-3.4$ & $3.5-4.5$ & $>4.5$ & \\
\hline $\operatorname{RRT}(n, \%)$ & $\begin{array}{l}57 \\
(17.8 \%)\end{array}$ & $7(9.0 \%)$ & $8(9.9 \%)$ & $\begin{array}{l}14 \\
(17.7 \%)\end{array}$ & $28(33.7 \%)$ & $<0.001$ \\
\hline LOS in hospital(day) & $\begin{array}{l}10.0(5.9- \\
18.2)\end{array}$ & $\begin{array}{l}8.8(5.6- \\
14.9)\end{array}$ & $\begin{array}{l}8.7(4.8- \\
16.4)\end{array}$ & $\begin{array}{l}10.7(6.3- \\
21.0)\end{array}$ & $\begin{array}{l}13.6(7.9- \\
23.1)\end{array}$ & 0.122 \\
\hline $\operatorname{AKI}(n, \%)$ & $\begin{array}{l}204 \\
(63.6 \%)\end{array}$ & $\begin{array}{l}41 \\
(52.6 \%)\end{array}$ & $46(56.8 \%)$ & $\begin{array}{l}54 \\
(68.4 \%)\end{array}$ & $63(75.9 \%)$ & 0.008 \\
\hline AKI Stage(n,\%) & & & & & & $<0.001$ \\
\hline 1 & $\begin{array}{l}46 \\
(22.5 \%)\end{array}$ & $\begin{array}{l}18 \\
(43.9 \%)\end{array}$ & $8(17.4 \%)$ & $\begin{array}{l}12 \\
(22.2 \%)\end{array}$ & $8(12.7 \%)$ & \\
\hline 2 & $\begin{array}{l}76 \\
(37.3 \%)\end{array}$ & $\begin{array}{l}13 \\
(31.7 \%)\end{array}$ & $26(56.5 \%)$ & $\begin{array}{l}21 \\
(38.9 \%)\end{array}$ & $16(25.4 \%)$ & \\
\hline 3 & $\begin{array}{l}82 \\
(40.2 \%)\end{array}$ & $\begin{array}{l}10 \\
(24.4 \%)\end{array}$ & $12(26.1 \%)$ & $\begin{array}{l}21 \\
(38.9 \%)\end{array}$ & $39(61.9 \%)$ & \\
\hline \multicolumn{7}{|l|}{ Lab parameters } \\
\hline $\mathrm{CK}(\mathrm{U} / \mathrm{L})$ & $\begin{array}{l}5143.0 \\
(1744.0- \\
15621.5)\end{array}$ & $\begin{array}{l}4543.5 \\
(1882.2- \\
12113.2)\end{array}$ & $\begin{array}{l}3725.0 \\
(1597.0- \\
10204.0)\end{array}$ & $\begin{array}{l}5832.0 \\
(1743.0- \\
15621.5)\end{array}$ & $\begin{array}{l}9140.0 \\
(2040.5- \\
32745.0)\end{array}$ & $<0.001$ \\
\hline $\mathrm{BUN}(\mathrm{mg} / \mathrm{dL})$ & $\begin{array}{l}24.0 \\
(15.0- \\
42.0)\end{array}$ & $\begin{array}{l}21.0 \\
(12.0- \\
30.8)\end{array}$ & $\begin{array}{l}18.0(13.0- \\
28.0)\end{array}$ & $\begin{array}{l}23.0(17.0- \\
33.0)\end{array}$ & $\begin{array}{l}56.0 \\
(29.5- \\
77.0)\end{array}$ & $<0.001$ \\
\hline $\operatorname{ALT}(\mathrm{U} / \mathrm{L})$ & $\begin{array}{l}179.0 \\
(52.0- \\
321.7)\end{array}$ & $\begin{array}{l}94.5 \\
(46.2- \\
321.7)\end{array}$ & $\begin{array}{l}176.0 \\
(43.0- \\
321.7)\end{array}$ & $\begin{array}{l}321.7 \\
(62.0- \\
321.7)\end{array}$ & $\begin{array}{l}196.0 \\
(67.0- \\
321.7)\end{array}$ & 0.055 \\
\hline $\mathrm{AST}(\mathrm{U} / \mathrm{L})$ & $\begin{array}{l}476.0 \\
(115.0- \\
626.0)\end{array}$ & $\begin{array}{l}221.0 \\
(98.8- \\
626.0)\end{array}$ & $\begin{array}{l}339.0 \\
(91.0- \\
626.0)\end{array}$ & $\begin{array}{l}626.0 \\
(148.0- \\
626.0)\end{array}$ & $\begin{array}{l}626.0 \\
(149.0- \\
779.5)\end{array}$ & 0.011 \\
\hline Platelet $\left(1000 / \mathrm{mm}^{3}\right)$ & $\begin{array}{l}201.4 \\
(126.0- \\
256.0)\end{array}$ & $\begin{array}{l}160.5 \\
(104.0- \\
224.0)\end{array}$ & $\begin{array}{l}228.0 \\
(139.0- \\
266.0)\end{array}$ & $\begin{array}{l}204.0 \\
(152.0- \\
246.0)\end{array}$ & $\begin{array}{l}195.0 \\
(123.0- \\
280.0)\end{array}$ & 0.012 \\
\hline $\mathrm{CR}(\mathrm{mg} / \mathrm{dL})$ & $2.0 \pm 1.9$ & $1.3 \pm 0.9$ & $1.3 \pm 1.0$ & $1.6 \pm 1.1$ & $3.8 \pm 2.7$ & $<0.001$ \\
\hline Bicarbonate $(\mathrm{mmol} / \mathrm{L})$ & $21.8 \pm 5.2$ & $22.5 \pm 5.7$ & $23.2 \pm 4.8$ & $22.1 \pm 4.5$ & $19.3 \pm 4.8$ & $<0.001$ \\
\hline HCT(\%) & $34.7 \pm 5.6$ & $34.7 \pm 5.3$ & $35.1 \pm 5.1$ & $35.7 \pm 5.8$ & $33.5 \pm 6.1$ & 0.09 \\
\hline \multicolumn{7}{|c|}{$\begin{array}{l}\text { AKI:acute kidney injury;CK:creatine kinase; CR:creatine; BUN:blood urea nitrogen; ALT:alanine } \\
\text { transaminase; AST:aspartate aminotransferase; HCT:hematocrit; HGB:hemoglobin; WBC:white blood } \\
\text { cell; RDW:red cell distribution width; AHF:acute heart failure; ALF:acute liver failure; AF:atrial } \\
\text { fibrillation; ARDS:acute respiratory distress syndrome; MBP:mean blood pressure (MBP); HR:heart } \\
\text { rate; RR:respiratory rate; RRT:renal replacement therapy; LOS:length of stay; SOFA:Sequential organ } \\
\text { failure assessment; APS区:acute physiology score区. }\end{array}$} \\
\hline
\end{tabular}




\begin{tabular}{|c|c|c|c|c|c|c|}
\hline \multirow[t]{2}{*}{ Characteristics } & \multirow[t]{2}{*}{ All } & \multicolumn{4}{|c|}{ phosphate levels (mg/dL) } & \multirow[t]{2}{*}{$\mathbf{P}$} \\
\hline & & $<2.6$ & 2.6-3.4 & $3.5-4.5$ & $>4.5$ & \\
\hline $\mathrm{HGB}(\mathrm{g} / \mathrm{dL})$ & $11.9 \pm 1.9$ & $11.9 \pm 1.9$ & $12.2 \pm 1.7$ & $12.2 \pm 2.0$ & $11.4 \pm 2.1$ & 0.043 \\
\hline Calcium(mg/dL) & $7.7 \pm 1.0$ & $7.4 \pm 1.0$ & $7.9 \pm 0.9$ & $7.8 \pm 0.8$ & $7.6 \pm 1.4$ & 0.005 \\
\hline Sodium(mmol/L) & $\begin{array}{l}139.5 \pm \\
6.7\end{array}$ & $\begin{array}{l}140.2 \pm \\
8.3\end{array}$ & $\begin{array}{l}140.0 \pm \\
6.4\end{array}$ & $\begin{array}{l}139.7 \pm \\
4.6\end{array}$ & $\begin{array}{l}138.1 \pm \\
7.0\end{array}$ & 0.173 \\
\hline Potassium(mmol/L) & $4.2 \pm 0.9$ & $3.6 \pm 0.7$ & $3.9 \pm 0.7$ & $4.4 \pm 0.7$ & $4.9 \pm 1.0$ & $<0.001$ \\
\hline Lactate (mmol/L) & $2.5 \pm 1.7$ & $2.4 \pm 1.4$ & $2.5 \pm 1.3$ & $2.3 \pm 1.1$ & $3.0 \pm 2.5$ & 0.039 \\
\hline $\operatorname{WBC}\left(10^{9} / \mathrm{L}\right)$ & $11.7 \pm 5.8$ & $10.6 \pm 5.7$ & $11.2 \pm 5.1$ & $12.4 \pm 5.6$ & $12.7 \pm 6.5$ & 0.085 \\
\hline RDW(\%) & $14.4 \pm 1.4$ & $14.1 \pm 1.4$ & $14.3 \pm 1.5$ & $14.5 \pm 1.3$ & $14.6 \pm 1.5$ & 0.103 \\
\hline \multicolumn{7}{|l|}{ Comorbidities,n(\%) } \\
\hline Hypertension & $\begin{array}{l}118 \\
(36.8 \%)\end{array}$ & $\begin{array}{l}21 \\
(26.9 \%)\end{array}$ & $\begin{array}{l}36 \\
(44.4 \%)\end{array}$ & $\begin{array}{l}28 \\
(35.4 \%)\end{array}$ & $\begin{array}{l}33 \\
(39.8 \%)\end{array}$ & 0.128 \\
\hline Diabetes & $\begin{array}{l}57 \\
(17.8 \%)\end{array}$ & $\begin{array}{l}12 \\
(15.4 \%)\end{array}$ & $\begin{array}{l}14 \\
(17.3 \%)\end{array}$ & $\begin{array}{l}11 \\
(13.9 \%)\end{array}$ & $\begin{array}{l}20 \\
(24.1 \%)\end{array}$ & 0.335 \\
\hline AHF & $24(7.5 \%)$ & $2(2.6 \%)$ & $\begin{array}{l}10 \\
(12.3 \%)\end{array}$ & $4(5.1 \%)$ & $8(9.6 \%)$ & 0.081 \\
\hline ALF & $\begin{array}{l}38 \\
(11.8 \%)\end{array}$ & $9(11.5 \%)$ & $7(8.6 \%)$ & $9(11.4 \%)$ & $\begin{array}{l}13 \\
(15.7 \%)\end{array}$ & 0.577 \\
\hline $\mathrm{AF}$ & $\begin{array}{l}42 \\
(13.1 \%)\end{array}$ & $4(5.1 \%)$ & $\begin{array}{l}17 \\
(21.0 \%)\end{array}$ & $9(11.4 \%)$ & $\begin{array}{l}12 \\
(14.5 \%)\end{array}$ & 0.028 \\
\hline ARDS & $4(1.2 \%)$ & $0(0.0 \%)$ & $1(1.2 \%)$ & $1(1.3 \%)$ & $2(2.4 \%)$ & 0.594 \\
\hline Stroke & $29(9.0 \%)$ & $5(6.4 \%)$ & $\begin{array}{l}13 \\
(16.0 \%)\end{array}$ & $5(6.3 \%)$ & $6(7.2 \%)$ & 0.088 \\
\hline \multicolumn{7}{|l|}{ Scoring systems } \\
\hline SOFA & $5.7 \pm 3.8$ & $5.1 \pm 3.8$ & $4.7 \pm 3.4$ & $5.4 \pm 3.8$ & $7.5 \pm 3.6$ & $<0.001$ \\
\hline APSD & $\begin{array}{l}52.7 \pm \\
23.2\end{array}$ & $\begin{array}{l}48.8 \pm \\
23.4\end{array}$ & $\begin{array}{l}47.5 \pm \\
21.7\end{array}$ & $\begin{array}{l}52.3 \pm \\
24.4\end{array}$ & $\begin{array}{l}61.9 \pm \\
20.8\end{array}$ & $<0.001$ \\
\hline \multicolumn{7}{|c|}{$\begin{array}{l}\text { AKI:acute kidney injury;CK:Creatine kinase; CR:creatine; BUN:blood urea nitrogen; ALT:alanine } \\
\text { transaminase; AST:aspartate aminotransferase; HCT:hematocrit; HGB:hemoglobin; WBC:white blood } \\
\text { cell; RDW:red cell distribution width; AHF:acute heart failure; ALF:acute liver failure; AF:atrial } \\
\text { fibrillation; ARDS:acute respiratory distress syndrome; MBP:mean blood pressure (MBP); HR:heart } \\
\text { rate; RR:respiratory rate; RRT:renal replacement therapy; LOS:Iength of stay; SOFA:Sequential organ } \\
\text { failure assessment; APS囚:acute physiology score冈. }\end{array}$} \\
\hline
\end{tabular}

Table2: Comparison between non-AKI and AKI groups 


\begin{tabular}{|c|c|c|c|}
\hline & Non-AKI & AKI & $\mathrm{P}$ \\
\hline $\mathrm{N}$ & 117 & 204 & \\
\hline Age(year) & $51.8 \pm 17.4$ & $54.9 \pm 16.5$ & 0.109 \\
\hline Gender(n,\%) & & & 0.087 \\
\hline male & $86(73.5 \%)$ & $131(64.2 \%)$ & \\
\hline female & $31(26.5 \%)$ & $73(35.8 \%)$ & \\
\hline Ethnicity(n,\%) & & & 0.118 \\
\hline asian & $2(1.7 \%)$ & $2(1.0 \%)$ & \\
\hline hispanci & $7(6.0 \%)$ & $4(2.0 \%)$ & \\
\hline black & $17(14.5 \%)$ & $25(12.3 \%)$ & \\
\hline white & $81(69.2 \%)$ & $140(68.6 \%)$ & \\
\hline other & $10(8.5 \%)$ & $33(16.2 \%)$ & \\
\hline Phosphate(mg/dL) & $3.4 \pm 1.6$ & $4.1 \pm 2.0$ & $<0.001$ \\
\hline $\mathrm{MAP}(\mathrm{mmHg})$ & $83.6 \pm 12.7$ & $81.1 \pm 11.8$ & 0.078 \\
\hline $\mathrm{HR}(/ \min )$ & $90.8 \pm 15.5$ & $93.9 \pm 15.2$ & 0.080 \\
\hline $\mathrm{RR}(/ \min )$ & $19.4 \pm 3.2$ & $19.9 \pm 4.1$ & 0.216 \\
\hline Temperature $\left({ }^{\circ} \mathrm{C}\right)$ & $37.1 \pm 0.6$ & $37.0 \pm 0.8$ & 0.096 \\
\hline SP02(\%) & $97.4 \pm 1.7$ & $96.6 \pm 4.0$ & 0.061 \\
\hline $\operatorname{RRT}(n, \%)$ & $5(4.3 \%)$ & $52(25.5 \%)$ & $<0.001$ \\
\hline LOS in hospital(day) & $11.6 \pm 15.0$ & $16.5 \pm 13.9$ & 0.003 \\
\hline $\mathrm{CK}(\mathrm{U} / \mathrm{L})$ & $4925.0(2023.0-13643.0)$ & $5705.5(1713.8-15621.5)$ & 0.075 \\
\hline $\mathrm{BUN}(\mathrm{mg} / \mathrm{dL})$ & $22.0(11.0-42.0)$ & $25.0(17.0-42.2)$ & 0.464 \\
\hline $\operatorname{ALT}(\mathrm{U} / \mathrm{L})$ & $106.0(44.0-321.7)$ & 239.5 (62.5-321.7) & 0.083 \\
\hline AST(U/L) & $269.0(115.0-626.0)$ & $624.5(120.0-626.0)$ & 0.055 \\
\hline Platelet $\left(1000 / \mathrm{mm}^{3}\right)$ & $205.0(145.0-253.0)$ & $198.0(122.0-257.2)$ & 0.374 \\
\hline $\mathrm{CR}(\mathrm{mg} / \mathrm{dL})$ & $1.1(0.8-2.1)$ & $1.3(0.9-2.9)$ & 0.014 \\
\hline Bicarbonate(mmol/L) & $22.1 \pm 5.1$ & $21.6 \pm 5.2$ & 0.361 \\
\hline НСТ(\%) & $34.2 \pm 5.5$ & $35.0 \pm 5.7$ & 0.242 \\
\hline $\mathrm{HGB}(\mathrm{g} / \mathrm{dL})$ & $11.9 \pm 1.8$ & $12.0 \pm 2.0$ & 0.703 \\
\hline
\end{tabular}




\begin{tabular}{|llll|}
\hline Calcium(mg/dL) & $7.8 \pm 0.8$ & $7.6 \pm 1.2$ & 0.135 \\
\hline Sodium(mmol/L) & $140.1 \pm 7.2$ & $139.2 \pm 6.4$ & 0.261 \\
\hline Potassium(mmol/L) & $4.0 \pm 0.8$ & $4.3 \pm 1.0$ & 0.005 \\
\hline Lactate(mmol/L) & $2.4 \pm 1.7$ & $2.6 \pm 1.7$ & 0.261 \\
\hline WBC(10\%/L) & $11.0 \pm 5.7$ & $12.1 \pm 5.9$ & 0.108 \\
\hline RDW(\%) & $14.3 \pm 1.4$ & $14.4 \pm 1.5$ & 0.711 \\
\hline Hypertension(n,\%) & $41(35.0 \%)$ & $77(37.7 \%)$ & 0.629 \\
\hline Diabetes(n,\%) & $22(18.8 \%)$ & $35(17.2 \%)$ & 0.710 \\
\hline AHF(n,\%) & $7(6.0 \%)$ & $17(8.3 \%)$ & 0.441 \\
\hline ALF(n,\%) & $10(8.5 \%)$ & $28(13.7 \%)$ & 0.167 \\
\hline AF(n,\%) & $9(7.7 \%)$ & $33(16.2 \%)$ & 0.030 \\
\hline ARDS(n,\%) & $0(0.0 \%)$ & $4(2.0 \%)$ & 0.127 \\
\hline Stroke(n,\%) & $6(5.1 \%)$ & $23(11.3 \%)$ & 0.064 \\
\hline SOFA & $4.2 \pm 2.8$ & $6.5 \pm 4.1$ & $<0.001$ \\
\hline APS & $43.9 \pm 16.7$ & $57.8 \pm 24.8$ & $<0.001$ \\
\hline
\end{tabular}

AKl:acute kidney injury;CK:creatine kinase; CR:creatine; BUN:blood urea nitrogen; ALT:alanine transaminase; AST:aspartate aminotransferase; HCT:hematocrit; HGB:hemoglobin; WBC:white blood cell; RDW:red cell distribution width; AHF:acute heart failure; ALF:acute liver failure; AF:atrial fibrillation; ARDS:acute respiratory distress syndrome; MBP:mean blood pressure (MBP); HR:heart rate; RR:respiratory rate; RRT:renal replacement therapy; LOS:length of stay; SOFA:Sequential organ failure assessment; APS囚:acute physiology score冈. 
Table 3

Comparison among patients according to AKI stage

\begin{tabular}{|c|c|c|c|c|}
\hline \multirow[t]{2}{*}{ Characteristics } & \multicolumn{3}{|l|}{ AKI } & \multirow[t]{2}{*}{$\mathbf{P}$} \\
\hline & Stage 1 & Stage 2 & Stage 3 & \\
\hline $\mathrm{N}$ & 46 & 76 & 82 & \\
\hline Age(year) & $52.6 \pm 16.0$ & $58.3 \pm 16.1$ & $53.1 \pm 16.9$ & 0.077 \\
\hline \multicolumn{4}{|l|}{ Gender(n,\%) } & 0.923 \\
\hline male & $29(63.0 \%)$ & $48(63.2 \%)$ & $54(65.9 \%)$ & \\
\hline female & $17(37.0 \%)$ & $28(36.8 \%)$ & $28(34.1 \%)$ & \\
\hline \multicolumn{4}{|l|}{ Ethnicity(n,\%) } & 0.851 \\
\hline asian & $1(2.2 \%)$ & $0(0.0 \%)$ & $1(1.2 \%)$ & \\
\hline hispanci & $5(10.9 \%)$ & $12(15.8 \%)$ & $8(9.8 \%)$ & \\
\hline black & $1(2.2 \%)$ & $1(1.3 \%)$ & $2(2.4 \%)$ & \\
\hline white & $6(13.0 \%)$ & $11(14.5 \%)$ & $16(19.5 \%)$ & \\
\hline other & $33(71.7 \%)$ & $52(68.4 \%)$ & $55(67.1 \%)$ & \\
\hline $\mathrm{MAP}(\mathrm{mmHg})$ & $82.4 \pm 11.3$ & $80.1 \pm 11.6$ & $81.3 \pm 12.2$ & 0.554 \\
\hline $\mathrm{HR}(/ \min )$ & $90.9 \pm 16.0$ & $93.7 \pm 13.5$ & $95.7 \pm 16.1$ & 0.23 \\
\hline $\mathrm{RR}(/ \min )$ & $97.4 \pm 1.8$ & $97.0 \pm 1.8$ & $95.9 \pm 5.8$ & 0.067 \\
\hline Temperature $\left({ }^{\circ} \mathrm{C}\right)$ & $37.0 \pm 0.7$ & $37.1 \pm 0.7$ & $36.9 \pm 0.8$ & 0.154 \\
\hline SP02(\%) & $97.4 \pm 1.8$ & $97.0 \pm 1.8$ & $95.9 \pm 5.8$ & 0.067 \\
\hline $\operatorname{RRT}(\mathrm{n}, \%)$ & $4(8.7 \%)$ & $7(9.2 \%)$ & $41(50.0 \%)$ & $<0.001$ \\
\hline LOS in hospital(day) & $11.9 \pm 10.5$ & $13.5 \pm 9.1$ & $21.8 \pm 17.3$ & $<0.001$ \\
\hline CK(U/L) & $\begin{array}{l}4254.5(2233.8- \\
15621.5)\end{array}$ & $\begin{array}{l}3499.0(1497.8- \\
10327.5)\end{array}$ & $\begin{array}{l}9285.5(1799.0- \\
33722.2)\end{array}$ & $<0.001$ \\
\hline $\mathrm{BUN}(\mathrm{mg} / \mathrm{dL})$ & $22.5(16.0-32.2)$ & $21.0(14.0-32.0)$ & $38.0(23.0-57.8)$ & $<0.001$ \\
\hline $\operatorname{ALT}(\mathrm{U} / \mathrm{L})$ & $321.7(56.5-321.7)$ & $120.5(41.5-321.7)$ & 321.7 (84.8-321.7) & 0.446 \\
\hline AST(U/L) & $577.5(102.2-626.0)$ & $310.0(98.5-626.0)$ & $626.0(187.0-895.8)$ & 0.155 \\
\hline \multicolumn{5}{|c|}{$\begin{array}{l}\text { AKI:acute kidney injury;CK:creatine kinase; CR:creatine; BUN:blood urea nitrogen; ALT:alanine } \\
\text { transaminase; AST:aspartate aminotransferase; HCT:hematocrit; HGB:hemoglobin; WBC:white blood } \\
\text { cell; RDW:red cell distribution width; AHF:acute heart failure; ALF:acute liver failure; AF:atrial } \\
\text { fibrillation; ARDS:acute respiratory distress syndrome; MBP:mean blood pressure (MBP); HR:heart } \\
\text { rate; RR:respiratory rate; RRT:renal replacement therapy; LOS:length of stay; SOFA:Sequential organ } \\
\text { failure assessment; APS®:acute physiology score冈. }\end{array}$} \\
\hline
\end{tabular}




\begin{tabular}{|c|c|c|c|c|}
\hline \multirow[t]{2}{*}{ Characteristics } & \multicolumn{3}{|l|}{ AKI } & \multirow[t]{2}{*}{$\mathbf{P}$} \\
\hline & Stage 1 & Stage 2 & Stage 3 & \\
\hline Platelet $\left(1000 / \mathrm{mm}^{3}\right)$ & $202.7(118.0-250.0)$ & $207.0(143.8-265.2)$ & $184.5(113.0-240.8)$ & 0.079 \\
\hline $\mathrm{CR}(\mathrm{mg} / \mathrm{dL})$ & $1.1(0.8-1.7)$ & $1.0(0.8-1.7)$ & $2.9(1.3-4.5)$ & $<0.001$ \\
\hline Bicarbonate(mmol/L) & $20.8 \pm 6.2$ & $22.8 \pm 3.7$ & $20.9 \pm 5.7$ & 0.04 \\
\hline НСТ(\%) & $35.3 \pm 5.3$ & $35.6 \pm 5.8$ & $34.4 \pm 5.8$ & 0.388 \\
\hline $\mathrm{HGB}(\mathrm{g} / \mathrm{dL})$ & $12.1 \pm 1.8$ & $12.1 \pm 2.0$ & $11.8 \pm 2.1$ & 0.514 \\
\hline Calcium(mg/dL) & $7.6 \pm 1.3$ & $7.9 \pm 1.0$ & $7.4 \pm 1.2$ & 0.034 \\
\hline Sodium(mmol/L) & $139.9 \pm 7.4$ & $139.1 \pm 6.6$ & $138.9 \pm 5.7$ & 0.669 \\
\hline Potassium(mmol/L) & $4.0 \pm 1.0$ & $4.2 \pm 0.8$ & $4.6 \pm 1.0$ & 0.004 \\
\hline Lactate (mmol/L) & $2.3 \pm 1.7$ & $2.6 \pm 1.5$ & $2.7 \pm 1.7$ & 0.419 \\
\hline $\operatorname{WBC}\left(10^{9} / \mathrm{L}\right)$ & $12.3 \pm 6.6$ & $12.4 \pm 6.1$ & $11.8 \pm 5.2$ & 0.834 \\
\hline RDW(\%) & $14.2 \pm 1.6$ & $14.3 \pm 1.4$ & $14.6 \pm 1.4$ & 0.218 \\
\hline Hypertension(n,\%) & $13(28.3 \%)$ & $36(47.4 \%)$ & $28(34.1 \%)$ & 0.074 \\
\hline Diabetes(n,\%) & $8(17.4 \%)$ & $16(21.1 \%)$ & $11(13.4 \%)$ & 0.445 \\
\hline $\operatorname{AHF}(n, \%)$ & $4(8.7 \%)$ & $6(7.9 \%)$ & $7(8.5 \%)$ & 0.984 \\
\hline $\operatorname{ALF}(\mathrm{n}, \%)$ & $4(8.7 \%)$ & $6(7.9 \%)$ & $18(22.0 \%)$ & 0.02 \\
\hline $\mathrm{AF}(\mathrm{n}, \%)$ & $2(4.3 \%)$ & $14(18.4 \%)$ & $17(20.7 \%)$ & 0.043 \\
\hline $\operatorname{ARDS}(\mathrm{n}, \%)$ & $0(0.0 \%)$ & $2(2.6 \%)$ & $2(2.4 \%)$ & 0.55 \\
\hline Stroke(n,\%) & $5(10.9 \%)$ & $11(14.5 \%)$ & 7 (8.5\%) & 0.497 \\
\hline SOFA & $4.9 \pm 2.9$ & $4.7 \pm 3.3$ & $9.1 \pm 4.0$ & $<0.001$ \\
\hline 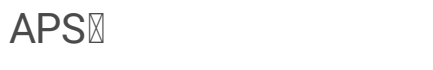 & $48.7 \pm 25.4$ & $47.3 \pm 16.2$ & $72.5 \pm 24.0$ & $<0.001$ \\
\hline \multicolumn{5}{|c|}{$\begin{array}{l}\text { AKI:acute kidney injury;CK:creatine kinase; CR:creatine; BUN:blood urea nitrogen; ALT:alanine } \\
\text { transaminase; AST:aspartate aminotransferase; HCT:hematocrit; HGB:hemoglobin; WBC:white blood } \\
\text { cell; RDW:red cell distribution width; AHF:acute heart failure; ALF:acute liver failure; AF:atrial } \\
\text { fibrillation; ARDS:acute respiratory distress syndrome; MBP:mean blood pressure (MBP); HR:heart } \\
\text { rate; RR:respiratory rate; RRT:renal replacement therapy; LOS:length of stay; SOFA:Sequential organ } \\
\text { failure assessment; APS囚:acute physiology score囚. }\end{array}$} \\
\hline
\end{tabular}

3.4 Risk of AKI and phosphate levels. We built a GAM model and multivariate logistic regression models to ascertain whether admission phosphate values contributed to AKI development. Group of phosphate $<2.6 \mathrm{mg} / \mathrm{dl}$ (lowest incidence of $\mathrm{AKI}, 52.6 \%$ ) was used as reference. The relationship between 
AKI and phosphate was linear after adjusting confounders and the smoothing spline plot showed a positive curve (Figure 3 ). The results of $P$ trend test were statistically significant according to phosphate levels, so there was no threshold saturation effect on the curve. To test this trend, both unadjusted and adjusted models were created. It was demonstrated that an increasing risk of AKI was associated with

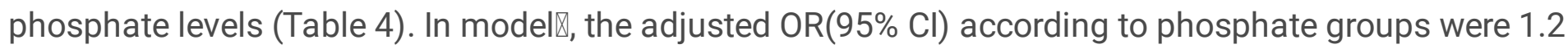
$(0.6,2.2), 1.9(1.0,3.7)$, and $2.8(1.4,5.5)$ respectively after adjusting for age and gender with $P$ trend value 0.001 in comparison with reference group. In model $\varangle$, after adjusting for age, gender, ethnicity, CK,

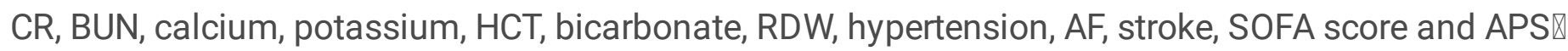
score, a similar trend was observed: the adjusted $\mathrm{OR}(95 \% \mathrm{Cl})$ were $1.1(0.5,2.4), 2.1(0.9,4.5), 2.7(1.0$, 7.4) respectively with $P$ trend value 0.034 .

Table 4

Association between risk of AKI and phosphate levels in different Logistic regression models

\begin{tabular}{|c|c|c|c|c|c|c|}
\hline & Non-adjusted & & Adjusted I & & Adjusted II & \\
\hline AKI & $\mathrm{OR}(95 \% \mathrm{Cl})$ & $P$ & $\mathrm{OR}(95 \% \mathrm{Cl})$ & $P$ & $\mathrm{OR}(95 \% \mathrm{Cl})$ & $\mathrm{P}$ \\
\hline Phosphate(mg/dL) & $1.3(1.1,1.4)$ & 0.001 & $1.3(1.1,1.4)$ & 0.001 & $1.3(1.1,1.6)$ & 0.012 \\
\hline \multicolumn{7}{|l|}{ Phosphate tertiles } \\
\hline$<2.6$ & 1.0 & & 1.0 & & 1.0 & \\
\hline $2.6-3.4$ & $1.2(0.6,2.2)$ & 0.593 & $1.2(0.6,2.2)$ & 0.654 & $1.1(0.5,2.4)$ & 0.733 \\
\hline $3.5-4.5$ & $1.9(1.0,3.7)$ & 0.044 & $1.9(1.0,3.7)$ & 0.049 & $2.1(0.9,4.5)$ & 0.074 \\
\hline$>4.5$ & $2.8(1.5,5.6)$ & 0.002 & $2.8(1.4,5.5)$ & 0.003 & $2.7(1.0,7.4)$ & 0.050 \\
\hline$P$ trend & 0.003 & & 0.001 & & 0.034 & \\
\hline \multicolumn{7}{|c|}{ Model囚adjusted for age and gender. } \\
\hline \multicolumn{7}{|c|}{ 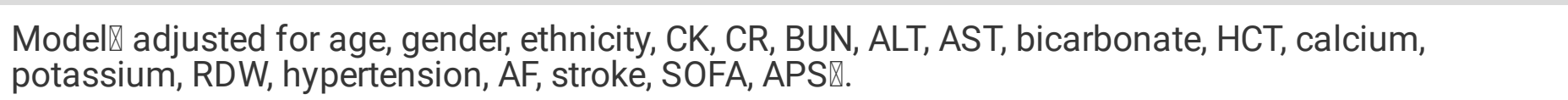 } \\
\hline \multicolumn{7}{|c|}{ OR:odds ratio; Cl:confidence interval; AKl:acute kidney injury. } \\
\hline
\end{tabular}

3.5 Subgroup Analyses. To further determine consistency of the relationship, subgroup analyses were performed according to dichotomous variables in patients with rhabdomyolysis. 30 variables were chosen as stratas and were divided into two equal groups (Table 5). CK was the only indicator where a statistically significant interaction was observed $(P=0.0196)$. Patients with $C K$ of more than $4952 \mathrm{U} / \mathrm{L}$ had a significantly higher risk of $\mathrm{AKI}(\mathrm{OR} 1.5,95 \% \mathrm{Cl} 1.2-1.8)$, whereas the other strata showed a relatively lower risk of AKI. 
Table 5

Subgroup analysis of the association between phosphate and risk of AKI

\begin{tabular}{|c|c|c|c|c|}
\hline Characteristic & $\mathbf{N}$ & OR (95\% Cl) & $\mathbf{P}$ & $P$ for interaction \\
\hline Age(year) & & & & 0.1491 \\
\hline$<54.4$ & 160 & $1.4(1.1,1.7)$ & 0.002 & \\
\hline$\geq 54.4$ & 161 & $1.1(0.9,1.4)$ & 0.218 & \\
\hline Gender(n,\%) & & & & 0.5891 \\
\hline male & 217 & $1.2(1.1,1.5)$ & 0.01 & \\
\hline female & 104 & $1.4(1.0,1.8)$ & 0.03 & \\
\hline $\mathrm{MAP}(\mathrm{mmHg})$ & & & & 0.7190 \\
\hline$<81.7$ & 150 & $1.2(1.0,1.5)$ & 0.037 & \\
\hline$\geq 81.7$ & 171 & $1.3(1.1,1.6)$ & 0.011 & \\
\hline $\mathrm{HR}(/ \min )$ & & & & 0.7002 \\
\hline$<92.4$ & 154 & $1.2(1.0,1.5)$ & 0.032 & \\
\hline$\geq 92.4$ & 167 & $1.4(1.1,1.7)$ & 0.007 & \\
\hline $\mathrm{RR}(/ \min )$ & & & & 0.6204 \\
\hline$<19.7$ & 152 & $1.2(1.0,1.5)$ & 0.055 & \\
\hline$\geq 19.7$ & 169 & $1.3(1.1,1.6)$ & 0.009 & \\
\hline Temperature $\left({ }^{\circ} \mathrm{C}\right)$ & & & & 0.064 \\
\hline$<37.0$ & 152 & $1.1(0.9,1.4)$ & 0.177 & \\
\hline$\geq 37.0$ & 169 & $1.5(1.2,1.9)$ & $<0.001$ & \\
\hline $\mathrm{SPO}_{2}(\%)$ & & & & 0.3585 \\
\hline$<97.1$ & 160 & $1.3(1.1,1.7)$ & 0.012 & \\
\hline$\geq 97.1$ & 161 & $1.2(1.0,1.4)$ & 0.025 & \\
\hline $\mathrm{CK}(\mathrm{U} / \mathrm{L})$ & & & & 0.0196 \\
\hline$<4952$ & 160 & $1.1(0.9,1.3)$ & 0.613 & \\
\hline
\end{tabular}

AKl:acute kidney injury;CK:creatine kinase; CR:creatine; BUN:blood urea nitrogen; ALT:alanine transaminase; AST:aspartate aminotransferase; HCT:hematocrit; HGB:hemoglobin; WBC:white blood cell; RDW:red cell distribution width; AHF:acute heart failure; ALF:acute liver failure; AF:atrial fibrillation; ARDS:acute respiratory distress syndrome; MBP:mean blood pressure (MBP); HR:heart rate; RR:respiratory rate; RRT:renal replacement therapy; SOFA:Sequential organ failure assessment; APS $₫:$ acute physiology score冈. 


\begin{tabular}{|c|c|c|c|c|}
\hline Characteristic & $\mathbf{N}$ & OR (95\% Cl) & $\mathbf{P}$ & $P$ for interaction \\
\hline$\geq 4952$ & 161 & $1.5(1.2,1.8)$ & $<0.001$ & \\
\hline $\mathrm{CR}(\mathrm{mg} / \mathrm{dL})$ & & & & 0.1440 \\
\hline$<1.3$ & 157 & $1.6(1.1,2.2)$ & 0.007 & \\
\hline$\geq 1.3$ & 164 & $1.2(1.0,1.4)$ & 0.026 & \\
\hline $\mathrm{BUN}(\mathrm{mg} / \mathrm{dL})$ & & & & 0.3675 \\
\hline$<24$ & 155 & $1.4(1.1,1.9)$ & 0.021 & \\
\hline$\geq 24$ & 166 & $1.2(1.0,1.4)$ & 0.016 & \\
\hline $\operatorname{ALT}(\mathrm{U} / \mathrm{L})$ & & & & 0.5729 \\
\hline$<179$ & 160 & $1.3(1.1,1.6)$ & 0.009 & \\
\hline$\geq 179$ & 161 & $1.2(1.0,1.5)$ & 0.063 & \\
\hline AST(U/L) & & & & 0.5714 \\
\hline$<476$ & 160 & $1.3(1.1,1.6)$ & 0.013 & \\
\hline$\geq 476$ & 161 & $1.2(1.0,1.4)$ & 0.065 & \\
\hline Bicarbonate $(\mathrm{mmol} / \mathrm{L})$ & & & & 0.2816 \\
\hline$<22$ & 151 & $1.2(1.0,1.4)$ & 0.03 & \\
\hline$\geq 22$ & 170 & $1.4(1.1,1.8)$ & 0.007 & \\
\hline НСТ(\%) & & & & 0.8492 \\
\hline$<34.6$ & 160 & $1.3(1.1,1.5)$ & 0.012 & \\
\hline$\geq 34.6$ & 161 & $1.3(1.0,1.6)$ & 0.026 & \\
\hline $\mathrm{HGB}(\mathrm{g} / \mathrm{dL})$ & & & & 0.1872 \\
\hline$<11.9$ & 152 & $1.2(1.0,1.4)$ & 0.033 & \\
\hline$\geq 11.9$ & 169 & $1.5(1.1,1.9)$ & 0.003 & \\
\hline Calcium(mg/dL) & & & & 0.0899 \\
\hline$<7.7$ & 154 & $1.2(1.0,1.4)$ & 0.059 & \\
\hline
\end{tabular}

AKI:acute kidney injury;CK:creatine kinase; CR:creatine; BUN:blood urea nitrogen; ALT:alanine transaminase; AST:aspartate aminotransferase; HCT:hematocrit; HGB:hemoglobin; WBC: white blood cell; RDW:red cell distribution width; AHF:acute heart failure; ALF:acute liver failure; AF:atrial fibrillation; ARDS:acute respiratory distress syndrome; MBP:mean blood pressure (MBP); HR:heart rate; RR:respiratory rate; RRT:renal replacement therapy; SOFA:Sequential organ failure assessment;

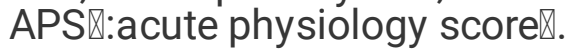




\begin{tabular}{|c|c|c|c|c|}
\hline Characteristic & $\mathbf{N}$ & OR (95\% Cl) & $\mathbf{P}$ & P for interaction \\
\hline$\geq 7.7$ & 167 & $1.5(1.2,2.0)$ & 0.002 & \\
\hline Sodium(mmol/L) & & & & 0.2152 \\
\hline$<140$ & 152 & $1.3(1.1,1.6)$ & 0.013 & \\
\hline$\geq 140$ & 169 & $1.2(1.0,1.4)$ & 0.094 & \\
\hline Potassium(mmol/L) & & & & 0.9634 \\
\hline$<4$ & 144 & $1.2(1.0,1.6)$ & 0.112 & \\
\hline$\geq 4$ & 177 & $1.2(1.0,1.5)$ & 0.034 & \\
\hline Lactate(mmol/L) & & & & 0.1242 \\
\hline$<2.5$ & 138 & $1.1(0.9,1.4)$ & 0.235 & \\
\hline$\geq 2.5$ & 183 & $1.4(1.1,1.7)$ & 0.001 & \\
\hline WBC $\left(10^{9} / \mathrm{L}\right)$ & & & & 0.7838 \\
\hline$<11$ & 159 & $1.3(1.1,1.5)$ & 0.009 & \\
\hline$\geq 11$ & 162 & $1.2(1.0,1.5)$ & 0.056 & \\
\hline Platelet $\left(1000 / \mathrm{mm}^{3}\right)$ & & & & 0.7075 \\
\hline$<201$ & 160 & $1.3(1.1,1.6)$ & 0.008 & \\
\hline$\geq 201$ & 161 & $1.2(1.0,1.5)$ & 0.048 & \\
\hline RDW(\%) & & & & 0.0032 \\
\hline$<14.1$ & 158 & $1.2(1.0,1.5)$ & 0.067 & \\
\hline$\geq 14.1$ & 163 & $1.3(1.1,1.6)$ & 0.005 & \\
\hline Hypertension(n,\%) & & & & 0.7464 \\
\hline No & 203 & $1.3(1.1,1.5)$ & 0.005 & \\
\hline Yes & 118 & $1.2(1.0,1.5)$ & 0.089 & \\
\hline Diabetes(n,\%) & & & & 0.0496 \\
\hline No & 264 & $1.3(1.1,1.5)$ & 0.001 & \\
\hline
\end{tabular}

AKI:acute kidney injury;CK:creatine kinase; CR:creatine; BUN:blood urea nitrogen; ALT:alanine transaminase; AST:aspartate aminotransferase; HCT:hematocrit; HGB:hemoglobin; WBC: white blood cell; RDW:red cell distribution width; AHF:acute heart failure; ALF:acute liver failure; AF:atrial fibrillation; ARDS:acute respiratory distress syndrome; MBP:mean blood pressure (MBP); HR:heart rate; RR:respiratory rate; RRT:renal replacement therapy; SOFA:Sequential organ failure assessment;

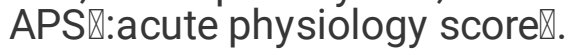




\begin{tabular}{|c|c|c|c|c|}
\hline Characteristic & $\mathbf{N}$ & OR (95\% Cl) & $\mathbf{P}$ & $P$ for interaction \\
\hline Yes & 57 & $1.1(0.8,1.5)$ & 0.388 & \\
\hline $\operatorname{AHF}(n, \%)$ & & & & 0.3117 \\
\hline No & 297 & $1.3(1.1,1.5)$ & $<0.001$ & \\
\hline Yes & 24 & $1.0(0.6,1.6)$ & 0.938 & \\
\hline $\operatorname{ALF}(n, \%)$ & & & & 0.5426 \\
\hline No & 283 & $1.3(1.1,1.5)$ & 0.001 & \\
\hline Yes & 38 & $1.1(0.8,1.5)$ & 0.646 & \\
\hline $\operatorname{AF}(n, \%)$ & & & & 0.9371 \\
\hline No & 279 & $1.3(1.1,1.4)$ & 0.002 & \\
\hline Yes & 42 & $1.3(0.7,2.3)$ & 0.392 & \\
\hline Stroke(n,\%) & & & & 0.3265 \\
\hline No & 292 & $1.2(1.1,1.4)$ & 0.002 & \\
\hline Yes & 29 & $1.9(0.7,4.8)$ & 0.179 & \\
\hline SOFA & & & & 0.4470 \\
\hline$<5$ & 140 & $1.3(1.0,1.7)$ & 0.052 & \\
\hline$\geq 5$ & 181 & $1.2(1.0,1.4)$ & 0.084 & \\
\hline APS & & & & 0.3169 \\
\hline$<51$ & 158 & $1.1(0.9,1.4)$ & 0.472 & \\
\hline$\geq 51$ & 163 & $1.3(1.0,1.5)$ & 0.022 & \\
\hline \multicolumn{5}{|c|}{$\begin{array}{l}\text { AKI:acute kidney injury;CK:creatine kinase; CR:creatine; BUN:blood urea nitrogen; ALT:alanine } \\
\text { transaminase; AST:aspartate aminotransferase; HCT:hematocrit; HGB:hemoglobin; WBC:white blood } \\
\text { cell; RDW:red cell distribution width; AHF:acute heart failure; ALF:acute liver failure; AF:atrial } \\
\text { fibrillation; ARDS:acute respiratory distress syndrome; MBP:mean blood pressure (MBP); HR:heart } \\
\text { rate; RR:respiratory rate; RRT:renal replacement therapy; SOFA:Sequential organ failure assessment; } \\
\text { APS囚:acute physiology score冈. }\end{array}$} \\
\hline
\end{tabular}

\section{Discussion}

In this study, we found a close relationship between phosphate values on admission and risk of AKI in patients with rhabdomyolysis in the ICU. Greater risk of AKI was associated with increasing phosphate levels. Patients with admission phosphate $₫ 4.5 \mathrm{mg} / \mathrm{dl}$ were found to have the highest incidence of AKI of total patients. After adjusting for potential covariables, the risk of AKI increased by 1.3 times in all 
patients and 2.7 fold in the group of the highest phosphate level in accordance with the lowest phosphate level group as reference.

The incidence of AKI varies among reports in patients with rhabdomyolysis. This may be due to the ambiguous definition of rhabdomyolysis and the different ways in which AKI is defined. In addition, the designs, target population and the etiology of rhabdomyolysis vary from study to study. As a result, these differences make the results more difficult to compare. In our study, we focused on the risk of AKI in ICU patients with rhabdomyolysis. AKI is characterized by rapid decline in kidney function in a relatively short time, which differs from chronic kidney disease(CKD). A multi-center retrospective study in France found an incidence of AKI of $81.4 \%$ in 387 patients with severe rhabdomyolysis from eight hospital ICU, even higher than $63.6 \%$ in ours[28]. However, the inclusion criteria of their study was a minimum serum CK level higher than $5000 \mathrm{U} / \mathrm{L}$ in $72 \mathrm{~h}$ following admission[28]. As is evident from our study, critically ill patients with rhabdomyolysis are at high risk of AKI. We also observed that the uptrend between AKI and phosphate levels during stage 3 was consistent with the overall patients uptrend while the trend of stage 1 and stage 2 was not stable. Because patients in stage 3 are more serious, the severity of AKI can be better reflected. In our study, CK, CR, BUN, bicarbonate, potassium values, SOFA score and APS $₫$ score are higher in stage 3 than in the other groups, so the trend of AKI incidence in stage 3 was more representative.

Phosphorus is present in cells throughout our body and is involved in almost all physiological and chemical reactions in the body[18]. Electrolyte and mineral imbalances are common in rhabdomyolysis patients. Disturbance of regulatory mechanism can lead to serious outcomes. This mechanism is more pronounced in patients admitted to the ICU, often with severe consequences. Elevated serum phosphate could lead to a range of diseases or increase the risk of diseases. One study analyzed 198 mechanical ventilation patients with severe sepsis or septic shock in ICU and found that time-weighted high serum phosphate increased patient mortality[29]. Charat Thongprayoon et al. found that elevated serum phosphate levels at admission can increase the risk of AKI[30]. In their grouping of phosphate $>4.4 \mathrm{mg} / \mathrm{dl}$, the $\mathrm{OR}(95 \% \mathrm{Cl})$ after multivariate logistic regression adjustment was $1.72(1.20,2.47)$, which showed that phosphate was a risk factor to the risk of AKI. In our results, in the phosphate $>4.5 \mathrm{mg} / \mathrm{dl}$ group, the adjusted result was $2.7(1.0,7.4)$, which was similar to the previous result.

The mechanisms responsible for the association between AKI and phosphate are not fully investigated yet. There are several possible explanations for these findings. Rhabdomyolysis and tumolysis syndrome (TLS) are important reasons of elevated phosphate and AKI[31]. Elimination of phosphate through kidney mainly depends on the renal threshold and the filtered load. Elevated levels of phosphate in plasma or renal tubules could lead to calcium-phosphate crystal deposition which bind to the tubular epithelial cells resulting in reactive oxygen damage[32,33]. Some suggest that it is the main pathway inducing renal impairment[32, 34]. If acute phosphate nephropathy(ATN) occurs during bowel preparation, it has been demonstrated that calcium-phosphate crystal deposition could cause damage to distal tubules and collecting ducts due[35]. Hypocalcemia due to elevated calcium-phosphate can be found in the condition of TLS and rhabdomyolysis[31, 36]. Our study found that with the increase of phosphate level, calcium 
level decreased, and the mean value of calcium $(7.7 \mathrm{mg} / \mathrm{dl})$ in this study was lower than the normal standard of $9-11 \mathrm{mg} / \mathrm{dl}$. The relationship needs further study and discussion.

Disruption of phosphate metabolism in rhabdomyolysis may be another path for calcium-phosphate deposition due to the phosphate retention. Rhabdomyolysis interferes with oxidative metabolism, which uses phosphate for Adenosine Triphosphate(ATP) production[37]. As a result, muscle damage in rhabdomyolysis causes impaired phosphate use and increases the phosphate load. Masato Higaki et al. considered that interference of phosphate use pathway contributes more significantly to the phosphate load rather than increased phosphate release in rhabdomyolysis[38]. The ATP depletion due to rhabdomyolysis affects the transport of phosphorus and calcium, which can exacerbate calciumphosphate deposition. Elevated phosphate levels have been found to increase fibroblast growth factor 23(FGF-23)[39]. Elevated FGF23 has been considered a strong predictor of adverse outcomes in patients with CKD and cardiovascular disease in this population[40]. With the further discovery of proinflammatory effects proved by upregulation of hepatic IL-6 production[41], profibrotic effects on the kidneys[42] and impairment of immune function[43], FGF23 is believed to have association with the risk of AKI and have prognostic utility in AKI[44].

In subgroup analysis, no significant interactions were observed except creatine kinase after patients were stratified according to binary grouping based on potential confounding factors. As is known to us, creatine kinase is released from damaged cells when rhabdomyolysis happens. Creatine kinase is also the diagnostic standard for rhabdomyolysis. The level of creatine kinase predicts the severity of rhabdomyolysis. A higher level of creatine kinase indicates severe rhabdomyolysis which is more likely to develop AKI. So the association between creatine kinase and phosphate in developing AKI needs more investigation.

Several limitations exist in this study. (1) Our study was a retrospective single-center study with a relatively homogenous population consisting mainly of white people. Therefore, further study of different ethnic groups is needed to determine the relationship between phosphate and AKI risk. (2) Although the association between the two was indicated, this study could not confirm that elevated serum phosphate level is the cause of AKI, which requires further prospective cohort studies or randomized controlled trial(RCT) studies. (3) Due to the limitations of the database, we could not extract more meaningful variable data, such as myoglobin, which has a more direct effect on the incidence of AKI, so there may be potential unadjusted pathogenic factors.

\section{Conclusions}

Serum phosphate level at admission was independently associated with an increased risk of acute kidney injury in critically ill patients with rhabdomyolysis. As phosphate levels rise, the risk of acute kidney injury increased.

\section{Abbreviations}


AKI

acute kidney injury

ICU

intensive care unit

MIMIC-区

Medical Information Mart for Intensive Care区

CK

creatine kinase

KDIGO

Kidney Disease Improving Global Outcome

APN

acute phosphate nephropathy

ICD-9

9th International Classification of Diseases

$\mathrm{CR}$

creatine

BUN

blood urea nitrogen

ALT

alanine transaminase

AST

aspartate aminotransferase

HCT

hematocrit

HGB

hemoglobin

WBC

white blood cell

RDW

red cell distribution width

AHF

acute heart failure

ALF

acute liver failure

AF

atrial fibrillation

ARDS

acute respiratory distress syndrome

MBP

mean blood pressure (MBP) 
HR

heart rate

RR

respiratory rate

RRT

renal replacement therapy

LOS

length of stay

SOFA

Sequential organ failure assessment

APS》

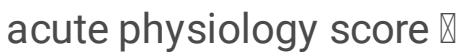

SD

standard deviation

IQR

inter quartile range

GAM

generalized additive model

OR

odds ratio

$\mathrm{Cl}$

confidence interval

TLS

tumolysis syndrome

ATP

Adenosine Triphosphate

FGF23

fibroblast growth factor 23

RCT

randomized controlled trial.

\section{Declarations}

Ethics approval and consent to participate

Not applicable.

Consent for publication

Not applicable.

Availability of data and materials 
The datasets used during the current study are available from the corresponding author upon reasonable request.

\section{Funding}

Innovation Research of Chinese PLA general hospital(CX19010).

Health care special research of Chinese PLA(20BJZ27)

\section{Competing interests}

The authors declare that they have no competing interests.

\section{Authors' contributions}

FHZ designed the idea of the article. TW, CL, ZM and XLW extracted data together from MIMIC- $\mathbb{X}$ database. TW and CL performed the data cleansing. TW and ZM analyzed the data. TW made the charts and finished the writing of the manuscript. All authors read and approved the final manuscript.

\section{Acknowledgements}

Ms. Jun Zhang assisted the author in writing and revising the manuscript.

\section{References}

1. Ahmad S, Anees M, Elahi I, Fazal-E-Mateen. Rhabdomyolysis Leading to Acute Kidney Injury. J Coll Physicians Surg Pak. 2021; 31(2):235-237.http://doi.org/10.29271/jcpsp.2021.02.235

2. Cabral BMI, Edding SN, Portocarrero JP, Lerma EV. Rhabdomyolysis. Disease-a-Month. 2020; 66(8):101015.http://doi.org/10.1016/j.disamonth.2020.101015

3. Zimmerman JL, Shen MC. Rhabdomyolysis. Chest. 2013; 144(3):10581065.http://doi.org/10.1378/chest.12-2016

4. Khan FY. Rhabdomyolysis: a review of the literature. Neth J Med. 2009; 67(9):272-283

5. Vanholder R, Sever MS, Erek E, Lameire N. Rhabdomyolysis. J Am Soc Nephrol. 2000; 11(8):15531561.http://doi.org/10.1681/ASN.V1181553

6. Haroun MW, Dieiev V, Kang J, Barbi M, Marashi NS, Gabr M, Eman G, Kajita G, Swedish K. Rhabdomyolysis in COVID-19 Patients: A Retrospective Observational Study. Cureus. 2021; 13(1):e12552.http://doi.org/10.7759/cureus.12552

7. Geng Y, Ma Q, Du YS, Peng N, Yang T, Zhang SY, Wu FF, Lin HL, Su L. Rhabdomyolysis is Associated with In-Hospital Mortality in Patients with COVID-19. Shock. 2021; 56(3):360367.http://doi.org/10.1097/SHK.0000000000001725

8. Zutt R, van der Kooi AJ, Linthorst GE, Wanders RJ, de Visser M. Rhabdomyolysis: review of the literature. Neuromuscul Disord. 2014; 24(8):651-659.http://doi.org/10.1016/j.nmd.2014.05.005 
9. Huerta-Alardín AL, Varon J, Marik PE. Bench-to-bedside review: Rhabdomyolysis -- an overview for clinicians. Crit Care. 2005; 9(2):158.http://doi.org/10.1186/cc2978

10. Simpson JP, Taylor A, Sudhan N, Menon DK, Lavinio A. Rhabdomyolysis and acute kidney injury: creatine kinase as a prognostic marker and validation of the McMahon Score in a 10-year cohort: A retrospective observational evaluation. Eur J Anaesthesiol. 2016; 33(12):906912.http://doi.org/10.1097/EJA.0000000000000490

11. Holt SG, Moore KP. Pathogenesis and treatment of renal dysfunction in rhabdomyolysis. Intensive Care Med. 2001; 27(5):803-811.http://doi.org/10.1007/s001340100878

12. Bagley WH, Yang H, Shah KH. Rhabdomyolysis. Intern Emerg Med. 2007; 2(3):210218.http://doi.org/10.1007/s11739-007-0060-8

13. Chavez LO, Leon M, Einav S, Varon J. Beyond muscle destruction: a systematic review of rhabdomyolysis for clinical practice. Crit Care. 2016; 20(1):135.http://doi.org/10.1186/s13054-016$1314-5$

14. Brun C, Munck O. Lesions of the kidney in acute renal failure following shock. Lancet. 1957; 272(6969):603-607.http://doi.org/10.1016/s0140-6736(57)91069-3

15. Chatzizisis YS, Misirli G, Hatzitolios Al, Giannoglou GD. The syndrome of rhabdomyolysis: complications and treatment. Eur J Intern Med. 2008; 19(8):568574.http://doi.org/10.1016/j.ejim.2007.06.037

16. Bosch X, Poch E, Grau JM. Rhabdomyolysis and acute kidney injury. N Engl J Med. 2009; 361(1):6272.http://doi.org/10.1056/NEJMra0801327

17. Subramanian R, Khardori R. Severe hypophosphatemia. Pathophysiologic implications, clinical presentations, and treatment. Medicine (Baltimore). 2000; 79(1):18.http://doi.org/10.1097/00005792-200001000-00001

18. Sim JJ, Bhandari SK, Smith N, Chung J, Liu IL, Jacobsen SJ, Kalantar-Zadeh K. Phosphorus and risk of renal failure in subjects with normal renal function. Am J Med. 2013; 126(4):311318.http://doi.org/10.1016/j.amjmed.2012.08.018

19. Foley RN. Phosphate levels and cardiovascular disease in the general population. Clin J Am Soc Nephrol. 2009; 4(6):1136-1139.http://doi.org/10.2215/CJN.01660309

20. Sullivan C, Sayre SS, Leon JB, Machekano R, Love TE, Porter D, Marbury M, Sehgal AR. Effect of food additives on hyperphosphatemia among patients with end-stage renal disease: a randomized controlled trial. JAMA. 2009; 301(6):629-635.http://doi.org/10.1001/jama.2009.96

21. Hruska KA, Mathew S, Lund R, Qiu P, Pratt R. Hyperphosphatemia of chronic kidney disease. Kidney Int. 2008; 74(2):148-157.http://doi.org/10.1038/ki.2008.130

22. Johnson AE, Pollard TJ, Shen L, Lehman LW, Feng M, Ghassemi M, Moody B, Szolovits P, Celi LA, Mark RG. MIMIC-III, a freely accessible critical care database. Sci Data. 2016; 3:160035.http://doi.org/10.1038/sdata.2016.35

23. Sauret JM, Marinides G, Wang GK. Rhabdomyolysis. Am Fam Physician. 2002; 65(5):907-912 
24. Stahl K, Rastelli E, Schoser B. A systematic review on the definition of rhabdomyolysis. J Neurol. 2020; 267(4):877-882.http://doi.org/10.1007/s00415-019-09185-4

25. Kellum JA, Lameire N. Diagnosis, evaluation, and management of acute kidney injury: a KDIGO summary (Part 1). Crit Care. 2013; 17(1):204.http://doi.org/10.1186/cc11454

26. Ostermann M, Bellomo R, Burdmann EA, Doi K, Endre ZH, Goldstein SL, Kane-Gill SL, Liu KD, Prowle JR, Shaw AD, et al. Controversies in acute kidney injury: conclusions from a Kidney Disease: Improving Global Outcomes (KDIGO) Conference. Kidney Int. 2020; 98(2):294309.http://doi.org/10.1016/j.kint.2020.04.020

27. Agoritsas T, Merglen A, Shah ND, O'Donnell M, Guyatt GH. Adjusted Analyses in Studies Addressing Therapy and Harm: Users' Guides to the Medical Literature. JAMA. 2017; 317(7):748759.http://doi.org/10.1001/jama.2016.20029

28. Candela N, Silva S, Georges B, Cartery C, Robert T, Moussi-Frances J, Rondeau E, Rebibou JM, Lavayssiere L, Belliere J, et al. Short- and long-term renal outcomes following severe rhabdomyolysis: a French multicenter retrospective study of 387 patients. Ann Intensive Care. 2020; 10(1):27.http://doi.org/10.1186/s13613-020-0645-1

29. Miller CJ, Doepker BA, Springer AN, Exline MC, Phillips G, Murphy CV. Impact of Serum Phosphate in Mechanically Ventilated Patients With Severe Sepsis and Septic Shock. J Intensive Care Med. 2020; 35(5):485-493.http://doi.org/10.1177/0885066618762753

30. Thongprayoon C, Cheungpasitporn W, Mao MA, Sakhuja A, Erickson SB. Admission hyperphosphatemia increases the risk of acute kidney injury in hospitalized patients. J Nephrol. 2018; 31(2):241-247.http://doi.org/10.1007/s40620-017-0442-6

31. Higaki M, Tanemoto $M$, Shiraishi T, Taniguchi K, Fujigaki Y, Uchida S. Acute Kidney Injury Facilitates Hypocalcemia by Exacerbating the Hyperphosphatemic Effect of Muscle Damage in Rhabdomyolysis. Nephron. 2015; 131(1):11-16.http://doi.org/10.1159/000437391

32. Lochy S, Jacobs R, Honore PM, De Waele E, Joannes-Boyau O, De Regt J, Van Gorp V, Spapen HD. Phosphate induced crystal acute kidney injury - an under-recognized cause of acute kidney injury potentially leading to chronic kidney disease: case report and review of the literature. Int $\mathrm{J}$ Nephrol Renovasc Dis. 2013; 6:61-64.http://doi.org/10.2147/IJNRD.S41428

33. Santos P, Branco A, Silva S, Paiva A, Baldaia J, Maximino J, Loureiro A, Henrique R. Acute phosphate nephropathy after bowel cleansing: still a menace. Nefrologia. 2010; 30(6):702704.http://doi.org/10.3265/Nefrologia.pre2010.Jul.10544

34. Slee TM, Vleming LJ, Valentijn RM. Renal failure due to acute phosphate nephropathy. Neth J Med. 2008; 66(10):438-441

35. Markowitz GS, Nasr SH, Klein P, Anderson H, Stack JI, Alterman L, Price B, Radhakrishnan J, D'Agati VD. Renal failure due to acute nephrocalcinosis following oral sodium phosphate bowel cleansing. Hum Pathol. 2004; 35(6):675-684.http://doi.org/10.1016/j.humpath.2003.12.005

36. Thongprayoon C, Cheungpasitporn W, Mao MA, Harrison AM, Erickson SB. Elevated admission serum calcium phosphate product as an independent risk factor for acute kidney injury in hospitalized 
patients. Hosp Pract (1995). 2019; 47(2):73-79.http://doi.org/10.1080/21548331.2019.1568719

37. Malis CD, Bonventre JV. Susceptibility of mitochondrial membranes to calcium and reactive oxygen species: implications for ischemic and toxic tissue damage. Prog Clin Biol Res. 1988; 282:235-259

38. Higaki M, Tanemoto $M$, Shiraishi T, Taniguchi K, Fujigaki Y, Uchida S. Acute Kidney Injury Facilitates Hypocalcemia by Exacerbating the Hyperphosphatemic Effect of Muscle Damage in

Rhabdomyolysis. Nephron. 2015; 131(1):11-16.http://doi.org/10.1159/000437391

39. Titan SM, Zatz R, Graciolli FG, Dos RL, Barros RT, Jorgetti V, Moyses RM. FGF-23 as a predictor of renal outcome in diabetic nephropathy. Clin J Am Soc Nephrol. 2011; 6(2):241247.http://doi.org/10.2215/CJN.04250510

40. Leaf DE, Siew ED, Eisenga MF, Singh K, Mc CF, Srivastava A, Ikizler TA, Ware LB, Ginde AA, Kellum JA, et al. Fibroblast Growth Factor 23 Associates with Death in Critically III Patients. Clin J Am Soc Nephrol. 2018; 13(4):531-541.http://doi.org/10.2215/CJN.10810917

41. Singh S, Grabner A, Yanucil C, Schramm K, Czaya B, Krick S, Czaja MJ, Bartz R, Abraham R, Di Marco GS, et al. Fibroblast growth factor 23 directly targets hepatocytes to promote inflammation in chronic kidney disease. Kidney Int. 2016; 90(5):985-996.http://doi.org/10.1016/j.kint.2016.05.019

42. Smith ER, Tan SJ, Holt SG, Hewitson TD. FGF23 is synthesised locally by renal tubules and activates injury-primed fibroblasts. Sci Rep. 2017; 7(1):3345.http://doi.org/10.1038/s41598-017-02709-w

43. Rossaint J, Oehmichen J, Van Aken H, Reuter S, Pavenstadt HJ, Meersch M, Unruh M, Zarbock A. FGF23 signaling impairs neutrophil recruitment and host defense during CKD. J Clin Invest. 2016; 126(3):962-974.http://doi.org/10.1172/JCl83470

44. Neyra JA, Hu MC, Moe OW. Fibroblast Growth Factor 23 and alphaKlotho in Acute Kidney Injury: Current Status in Diagnostic and Therapeutic Applications. Nephron. 2020; 144(12):665672.http://doi.org/10.1159/000509856

\section{Figures}


Patients diagnosed as rhabdomyolysis according to ICD-9 codes from MIMIC-II $(n=390)$

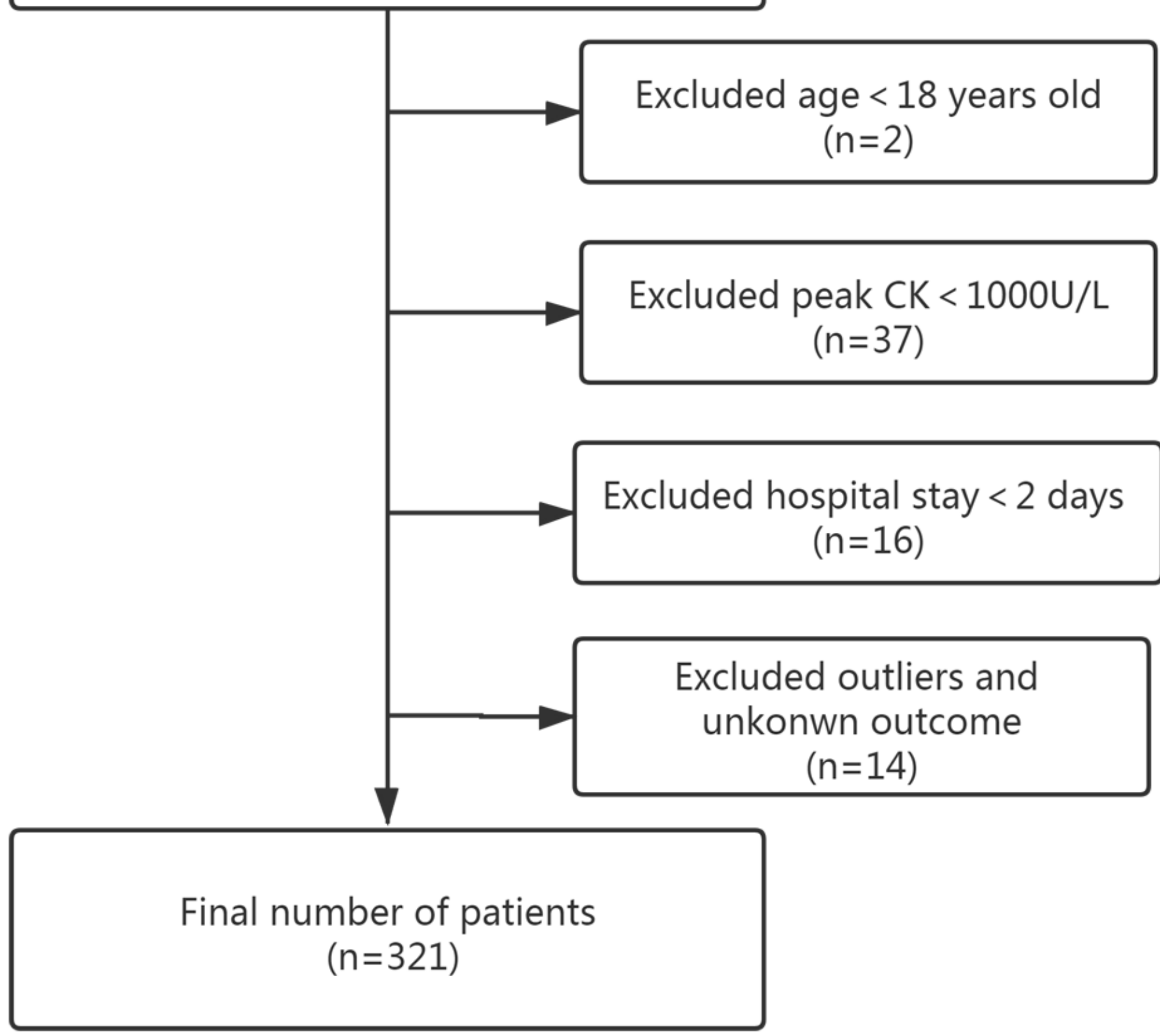

Figure 1

Flow chart of the screening process. 321 patients were finally selected. CK creatine kinase, ICD-9 International Classification of Diseases-9. 


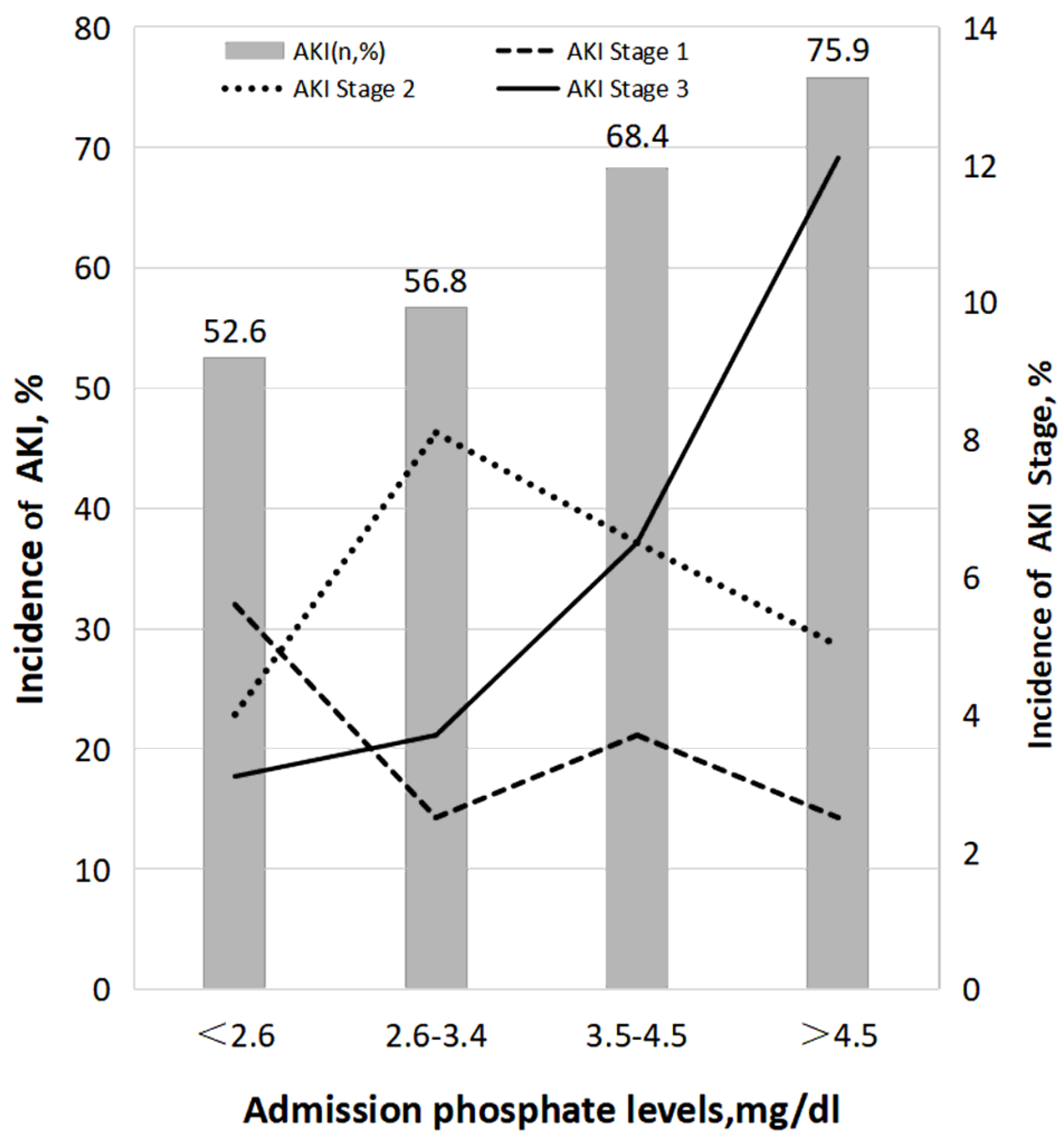

Figure 2

Comparison of incidence of AKI and AKI Stages among admission phosphate levels. AKI (\%) was represented by histograms and referenced to the left Y-axis. AKI stages (\%) were represented by line chart and referenced to the right Y-axis. Incidence of AKI in total patients increased with the elevation of phosphate levels. The same trend was observed in the stage 3. 


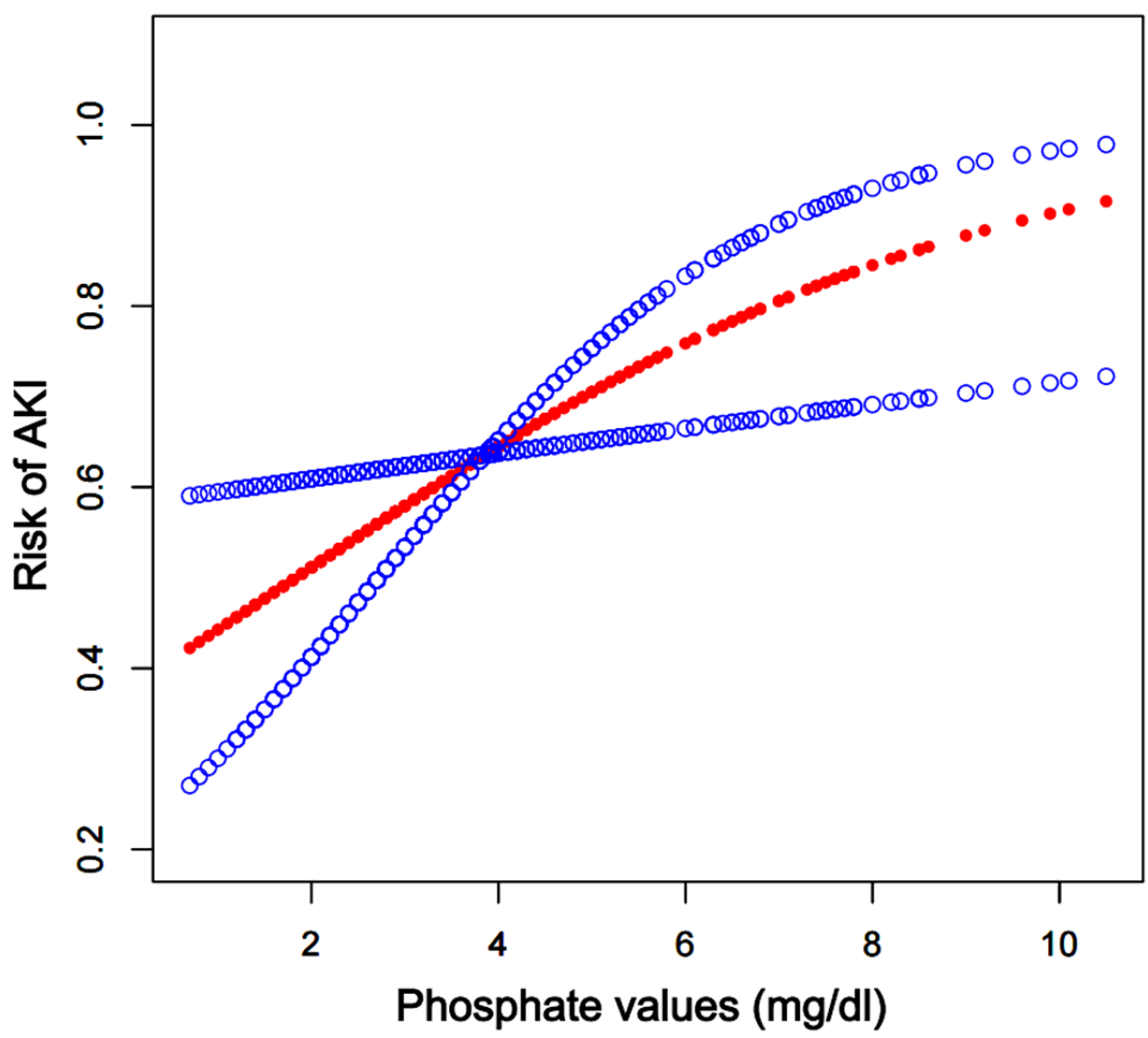

Figure 3

The nonlinear relationship by GAM between phosphate and risk of AKI. Red dotted lines represented the smooth curve fit. Blue dotted lines represented the 95 percent confidence interval from the fit. The models were adjusted for age,gender, ethnicity, CK, CR, BUN, ALT, AST, bicarbonate, HCT, calcium, potassium, RDW, hypertension, AF, stroke, SOFA, APS囚. GAM generalized additive model. 